\title{
评述
}

\section{大数据与城市生态学的未来: 从概念到结果}

\author{
杨军 ${ }^{1,2^{*}}$
}

1. 地球系统数值模拟教育部重点实验室, 清华大学地球系统科学系, 北京 100084 ;

2. 清华大学中国城市研究院, 北京 100084

* 通讯作者, E-mail: larix@tsinghua.edu.cn

收稿日期：2020-04-01; 收修改稿日期：2020-06-23; 接受日期：2020-07-27; 网络版发表日期：2020-08-14 国家重点研发计划项目(编号: 2019YFA0607201)资助

\begin{abstract}
摘要 城市生态学正在经历第三次研究范式的转变, 认识构成城市的社会系统和自然系统在时间和空间上跨尺 度的相互作用, 为城市可持续发展提供理论基础和解决方案将是城市生态学在未来十年的研究重点. 基于城市生 态学所具有的自然科学和社会科学高度交叉的属性, 城市是各种大数据的生产中心的现实, 以及在研究中使用大 数据后可获得的对城市生态系统的新认识, 可以预期大数据将在未来城市生态学研究中发挥重要的作用。但是, 要将大数据从一个概念转化成为能够指导城市规划和决策管理的城市生态学研究成果, 还需要克服在理论框 架、数据获取, 以及分析技术上的多重挑战. 城市生态学研究人员未来需要加强和数据科学家的合作, 在城市生 物多样性、城市生态系统服务和健康福祉、城市生态系统过程等研究方向上增加大数据的应用.
\end{abstract}

关键词 大数据, 社交媒体, 研究范式, 可持续发展, 城市生态系统

\section{1 引言}

城市化是对地球系统影响最大的人类活动之一, 仅占全球表面积 $3 \%$ 的城市区域消耗了全球生产的 $2 / 3$ 的初级能源, 释放的 $\mathrm{CO}_{2}$ 占到全球能源部门 $\mathrm{CO}_{2}$ 排放的 70\%(International Energy Agency, 2016). 在今后很长一 段时间内全球的城市化还将持续进行. 根据联合国估 计，到2050年全球城市人口占总人口的比例将从现在 的 55\%提升到68\%(United Nations, 2019). 城市的可持 续发展已经成为 21 世纪做关人类未来的基本问题.

在此背景下，城市被视为是测试未来人类和生态 系统如何和谐共生的实验室(Barot等, 2019). 作为研 究城市生态系统与人类社会之间的相互关系的学科,
城市生态学担负着为这项宏大的测试提供理论、方法 和问题的解决方案的重任. 为了应对这一需求, 城市生 态学研究在过去 20 年里发生了众多的变化. 当前城市 生态学正在经历研究范式上的转变. 这个转变体现在 城市生态学的主要研究问题、研究方法和研究目的 上. 城市生态学从关注城市内部的生态系统成分的结 构和动态变化, 转向关注构成城市的社会经济系统和 自然生态系统在跨时空尺度上的相互作用; 从使用传 统生态学研究方法为主, 转向以多学科和跨学科的方 法为主; 研究的目的也从以增加对城市生态系统的科 学认识为主发展到获得新认识和指导城市规划和管理 实践并重, 从而促进城市的可持续发展.

大数据科学的兴起可为城市生态学的发展提供一 org/10.1007/s11430-020-9666-3 
个关键的助力. 在人口在城市中的高度聚集, 以城市为 中心的经济生产方式和飞速扩张的网络、通信和物联 网的影响下，城市已经变成了一个巨大的数据工厂 (Economist，2012). 通过传感器、智能装置、网络和 社交媒体等现代信息科技捕获或记录的数字化信息构 成了城市大数据的主体. 城市大数据不仅能够帮助城 市生态学家实现对城市中自然生态系统的高时空分辨 率的监测, 其提供的数以亿计的个人行为的精细尺度 数据使得对个人到整个社会的跨尺度研究成为可能 (Rabari和Storper, 2015). 基于以上的特性, 在城市生态 研究中大数据的应用日益增加, 已被用于刻画城市生 态系统结构、监测城市生态系统过程、城市生物多样 性和量化城市生态系统服务及其对居民的健康福祉影 响等多个方面. 但是, 大数据方法要成为城市生态学的 主流研究方法还面临着诸多挑战. 本文介绍了城市生 态学的发展, 大数据在城市生态学发展中的潜在作用, 并回顾了在城市生态研究中大数据的应用现状、面临 的挑战和问题, 最后指出了在未来城市生态学研究中 大数据的重点应用方向.

\section{2 城市生态学的发展}

\section{1 城市生态学的历史发展}

城市生态学的前身可以追溯到在16世纪兴起的城 市植物学, 其以描述城市内部的植物和生境为主要研 究内容(Sukopp, 2008). 受这一起源影响, 城市生态学 研究在很长一段时间都遵循“城市中的生态学(Ecology in the City)”研究范式, 以城市中的非人类生物及 其生境为主要研究对象, 分析城市化对它们的影响. 研 究的主要目的是增加生态学认识和促进对城市中的生 物及生境的保护. 在研究方法上, 通常直接使用自然生 态研究中的方法或对其进行一定程度的修改后在城市 中应用. 这方面经典的例子包括生态单元制图法(Sukopp和Weiler，1988)和梯度分析方法(McDonnell和 Pickett，1990)在城市生态研究中的应用. 但即使是以 在城市中开展传统生态学研究作为主流方向的时期, 一些城市生态学者也在关注城市中社会系统和自然系 统的相互作用, 如城市生态学中芝加哥学派的代表性 人物Robert E. Park提出了同心圆理论, 很好地解释了 城市社会经济发展引起的城市土地利用的空间格局变 化(Park等, 1925).
自20世纪90年代后期开始，“城市的生态学(Ecology of the City)”逐渐发展成为了城市生态学的主流 研究范式. 该范式关注城市作为一个人与自然耦合的 生态系统, 在内部系统成分的相互作用和全球环境变 化影响下, 其结构和功能发生的变化(Grimm等, 2008). 在美国巴尔的摩市和菲尼克斯市进行的城市长期定位 生态研究是这一时期的代表性研究工作. 这些研究在 认识城市生态系统的空间复杂性、组织复杂性和时间 复杂性上持续地取得进展, 形成了城市生态学中的巴 尔的摩学派, 发展了包括源或汇、多层级斑块动态、 复合群落、动态异质性和城市同质化等多个理论 (Pickett等, 2020). 城市生态学的三个核心问题也在这 一时期被提出来(Young和Wolf, 2006): (1) 城市生态系 统是如何运转的? (2) 众多的影响因素如何影响城市 生态系统? (3) 如何用这些知识来解决当前的城市和 环境问题?

在进入 21 世纪以来, 随着以气候变化为代表的全 球变化对人类社会和环境影响的加剧, 可持续发展已 成为国际社会共同关注的核心问题. 作为全球人口高 度聚集的区域、资源和能源消费的中心，以及社会经 济、科技研发活动进行的主要场所, 城市能否实现可 持续发展决定着全球可持续发展的实现. 在这一背景 下，一些城市生态学家提出城市生态学应该呼应时代 的需求, 向“为了城市的生态学(Ecology for the City)” 的研究范式转变(Childers等, 2015). 城市生态学应关注 在跨单个城市、区域和全球尺度上的生态过程和社会 动态对城市的可持续性的影响. $\mathrm{Wu}(2014)$ 更是明确提 出, 当前是城市生态学的黄金时期, 城市生态应该在 全球可持续发展中扮演重要的角色.

总体来说, 城市生态学在认识城市化的格局、动 态及其生态影响上已经取得了显著的进步. 但是, 基 于城市是一个复杂的人和自然的耦合系统的认识之上 的、系统的城市生态科学还没有被完全建立起来. 对 城市这一复杂系统在很多方面的认识都还存在局限, 例如, Alberti(2017)曾提出: 城市区域的社会经济和生 态系统之间如何相互作用? 城市动态如何与社会生态 的变化以及城市中的进化机制相联系? 城市生态研究 如何和城市管理和规划的更好地结合? 这些问题都还 没有被很好地回答. 由于上述原因, 城市生态学没有获 得应有的重视. Gandy(2015)指出, 城市生态学还是被 视为生物学的一个下属学科或进行政治动员时使用的 
一个概念.

需要特别指出的是，中国的城市生态学发展受到 中国独特的城市化过程的影响. 与美国注重基本生态 学问题的研究不同, 中国的城市生态学研究比较注重 解决发展和环境变化中的实际问题(Lin和Grimm, 2014). 在1984年中国学者就已经提出了社会-经济-自 然复合系统的概念(马世俊和王如松, 1984), 这一概念 和“为了城市的生态学”的研究范式的核心观点非常接 近. 当前中国的城市生态学在发展中也同样面临着前 面文中列举的问题, 包括: 缺乏对城市生态学基础理 论的研究, 和城市规划基本上没有互动, 研究局限于 单个城市和区域, 以及缺乏多尺度的研究等 $(\mathrm{Wu}$ 等, 2014).

\section{2 城市生态学未来发展方向}

城市生态学要发展成一门系统科学, 并在支持全 球可持续发展中发挥其应有的作用, 在未来需要加强 三个方向上的研究: (1) 发展城市生态学的一般理论, (2) 加深对城市生态系统与人类福祉之间关系的理解, （3）加强城市生态研究对城市管理决策和规划设计的 支撑作用.

从单个城市到全球的尺度去总结一般规律，提出 城市生态学的基础理论, 是建立统一的城市生态科学 的前提. 城市被认为是一个新型生态系统(Novel ecosystem), 其包含着很多独特的环境和生物组合, 受到 人类活动的强烈影响. 因此, 基于自然生态系统发展 起来的生态学中的理论在多大程度上能够适用于城市 生态系统值得商権(Pataki, 2015). 与此同时, 由于城市 化模式的多样性, 在单个城市中获得的研究发现在更 大的空间尺度上是否成立也是一个经常被提出的问 题. 例如, 发达国家和发展中国家不同的城市化过程导 致城市结构上的不同，进而导致了城市化对生物多样 性和生态系统功能的影响上的差异(Barot等, 2019). 因而, 城市生态的研究一方面需要跨越多个时空尺度. 这一点在Pickett等(2020)提出的城市生态学未来应该 重点发展的复合城市、城市超级区域和城市性连续体 三个前沿理论中体现得非常明确. 复合城市理论认为 任何尺度的城市都是一个变化的、由生物物理环境、 人类社会、经济和政治结构，以及建成结构形成的镶 嵌体. 而城市超级区域理论认为城市是城市群的一部 分. 城市性连续体理论则更进一步, 认为全球是一个
城市性的连续体, 乡村和野外的土地和生物是和城市 里的土地和生物密切交织在一起的. 另一方面, 城市 生态的研究需要注重机制性模型的提出. 将城市大小 与城市增长的空间格局和经济过程联系在一起的尺度 定律(McDonald, 2016)就是一个成功的例子.

未来城市生态研究中的一个重要方向是理解城市 生态系统和人类福祉之间的关系. 人类建设城市的初 衰是保护人身和财物的安全，提供交易的场所，从而 增加人类福祉. 在城市出现的 6000 多年历史里, 虽然 城市的形态、结构、规模等发生了很大的变化, 但城 市增加人类福祉的根本作用没有发生改变. 城市居民 的福祉受到城市生物多样性、城市中社会系统和自然 系统的过程，以及它们产生的生态系统服务在跨空间 和时间尺度上的相互作用的影响. 因此, 要促进城市 的可持续性, 需要理解这些成分在关键的时空尺度上 的相互作用 (Pataki, 2015). 而为了理解这个相互作用, 需要回答一些关键问题, 例如, 城市中的社会经济成分 和自然成分如何共同产生生态系统服务? 如何权衡优 化各种生态系统服务从而提升人类福祉?

实现城市的可持续发展是建立在对城市的有效管 理的基础之上的. 但如果缺乏对高度复杂的、异质性 的城市生态系统的综合认识, 决策者将难以有效地管 理城市. 因此, 未来的城市生态学研究在增加对城市 生态系统的科学认识的同时, 必须促进研究和决策管 理、城市规划的结合(Tanner等, 2014). 这需要城市生 态学把回答和政策相关的问题也作为主要的研究目标 之一(Young和Wolf, 2006), 需要通过为决策提供工具 和当地的数据, 采用基于城市生态学知识和可持续性 原则的规划设计, 来影响或指引城市往有利于增加可 持续性的方向上发展 $(\mathrm{Wu}, 2014)$. 城市生态学研究和 规划管理的结合将是一个循环往复和不断递进的过 程, 城市生态学研究要有充分的科学性和严谨性来支 撑这个过程.

\section{3 大数据在城市生态学中的应用潜力与应 用现状}

\section{1 大数据在城市生态学中的应用潜力}

城市生态学在发展成为一个自然科学和社会科学 高度交叉、综合的城市系统科学的过程中需要新的理 论、方法和数据, 包括但不仅限于: 需要发展新方法、 
新工具来认识城市环境的复杂性( McPhearson等, 2016), 理解城市中的人与非人生物和环境间的相互作 用、反馈和非线性的过程; 需要在研究基于个体尺度 上的过程和分析生态系统尺度上的综合环境影响之间 取得平衡(Shochat等, 2006); 需要发展能将高度集中于 个体的尺度上的研究发现转化为大尺度的城市设计、 管理和政策提供科学支持的理论和模型(Pataki, 2015).

大数据科学为满足上述需求提供了可行的方法. 大数据在生态学研究中具有增加在大尺度上的科学理 解，促进高分辨率、多尺度和跨尺度的格局分析的潜 力(Durden等, 2017). 传感器网络监测数据、遥感数 据、社交媒体数据和城市管理数据能够提供高时空分 辨率的城市生态系统状态信息，提升识别城市生态系 统的复杂结构及其在时空上的动态变化的能力(Rabari 和Storper, 2015). WIFI热点接入、手机轨迹大数据能 够显示城市内外居民的活动信息，为评估居民如何使 用和响应城市中的自然系统提供了一个途径 (McPhearson等, 2016). 与通过传统的社会调查获得的 数据相比, 大数据具有样本量大、时空解析度高的优 点; 同时由于大数据通常是自动和被动收集的, 减少 了由于社会调查返回率低和受访者不真实回应所带来 的偏差(Lenormand和Ramasco, 2016; Wang和Moriarty, 2018). 因此, McPhearson等(2016)认为大数据的应用将 给城市社会生态系统的研究带来革命性的变化.

与此同时, 城市的规划、设计和决策管理将从大 数据中获益. 大数据将城市居民和周边环境的相互作 用和关系变得可视、可读, 从而变得可以管理(Shirtcliff，2015). 在当前各城市纷纷采用基于自然的解决 方案来应对面临的生态环境挑战时, 大数据能让决策 者将数量化的信息和其他类型的知识联系起来，让基 于自然的解决方案更适合当地的情况和需求(Frantzeskaki等, 2019; Ilieva和McPhearson, 2018).

\section{2 大数据在刻画城市生态系统结构中的应用}

城市产生的大数据为识别社会系统、自然系统的 结构, 以及它们在时间和空间上的位置创造了条件. 目 前的研究主要集中在应用地理空间大数据识别城市的 功能区, 结合地理空间大数据和遥感数据进行城市土 地覆盖/利用的分类, 以及基于街景等图片数据识别城 市中的建筑和街道、城市植被等构成要素等方面.

一些研究使用单一类型的大数据来识别城市的功
能区. 在智利, 研究者使用了 3 亿个手机接入数据, 分析 了全国 10 万人以上城市的个人行踪轨迹，通过识别高 度重合的区域和其在城市的功能上的重要性, 确定了 城市的边界(Sotomayor-Gómez和Samaniego, 2020). 在 一项对欧美 10 个主要城市的研究中, 研究者使用从Picaso和Flickr等社交媒体收集的7500万张有地理标记 和时间翟的照片，分析了城市居民和访客随时间变化 的空间分布, 发现了访客和居民活动的不同时间尺度, 以及美国和欧洲城市人群的活动高峰区在时间上的变 化差异(Paldino等, 2016). Boeing(2019)利用OpenStreetMap数据, 分析了全球 25 个城市的街道格局、走 向和空间的组成方式, 为城市形态研究提供了新的 思路.

但越来越多的研究倾向于综合使用多种类型的大 数据来识别城市的功能区. 如Cai等(2017)首先用微博 签到信息和夜间灯光卫星数据, 勾画了北京、上海和 重庆的城市中心区范围, 然后使用POI(Point of interest)数据对识别的精度和准确度进行了验证, 发现识别 准确度能达到 $80 \%$ 左右. 除了识别传统意义上的城市 功能区外, 一些研究还识别了对于城市规划和管理而 言具有意义的社会空间结构. 如在新加坡, $\mathrm{Xu}$ 等 (2019)使用手机轨迹数据, 结合社会经济数据, 分析了 居民在社会经济空间上的社会隔离和物理空间上的物 理隔离之间的关系, 证实新加坡存在着穷人和富人在 居住上的隔离状态. 刘瑜等(2018)采用城管执法数 据、POI数据、出租车上下车点数据和地铁出行距离 数据, 借用生态位模型的概念, 分析了城管执法事件的 潜在发生区域, 并和采用百度街景数据获得的城市环 境状况的分析结果进行对比, 其结果显示城管执法高 发的区域和城市的脏乱差区域是高度吻合的.

城市土地利用具有社会经济和生物物理两方面的 特性. 地理空间大数据在挖掘人的行为动态和社会经 济特征方面具有优势, 尤其是其中的社会感知大数据 提供了对人与环境之间关系的新认识(Liu等，2015). 遥感大数据能提供城市地表的生物物理特征信息, 如高分辨率遥感影像能够提供城市内部的详细信息, 包括城市建筑、道路、土地覆盖等(Gong J等, 2020; Huang等, 2020). 两种大数据的结合使用能够提高对 土地利用格局的认知. $\mathrm{Hu}$ 等(2016)使用POI数据、 OpenStreetMap数据和美国陆地卫星数据, 建立了针对 城市地块进行土地利用精细分类的方法, 生成了北京 
的城市土地利用图. 这个方向上的研究目前在继续深 入, 包括引入更多类型的社会经济大数据(Zhang等, 2020), 使用更复杂的算法(Cao等, 2020), 研究的尺度 也从单个城市发展到区域或国家尺度(Gong P 等, 2020). 地理空间大数据和遥感数据的结合不仅提高了 城市土地利用的识别效率, 还为将城市的社会经济数 据空间化提供了新的方法. 如Ye等(2019)结合应用POI 数据和多源遥感数据, 将中国 2010 年县级尺度的人口 普查数据分解到 $100 \mathrm{~m}$ 网格上, 其结果比基于通常的地 理数据、遥感产品和人口数据生成的WorldPop产品精 度更高.

受益于深度学习在图像识别中的应用进展, 基于 街景等图片数据来获得城市内部结构信息成为了城市 大数据分析的热点研究方向, 也为城市生态研究提供 了常规方法难以获取的数据. 如Li等(2015)使用Google 街景照片, 基于图像差分方法, 量化了街道尺度上 的视域范围内绿色植被所占的比例——绿视率. 该方 法与之前基于手工分析拍摄的照片的方法(Yang等, 2009)相比更准确、效率更高. 目前使用深度学习方法 来挖掘街景照片中的社会和自然系统信息的研究发展 迅猛. 如Cai等(2020)使用深度卷积神经网络, 结合标记 数据和Google街景数据, 将城市的绿色成分识别的平 均绝对错误从 $10.04 \%$ 降低到了 $4.67 \%$. Middel等(2019) 发展了基于可扩展的深度学习框架分割Google街景照 片并投影到一个球面上的方法, 来近似模拟行人在街 上的视角, 从而将分析的结果和人的活动、人体户外 热舒适度乃至野生动物的活动联系在一起. 街景照片 还被城市生态学者用于分析城市绿化与健康的关系 (Helbich等, 2019), 识别城市中生物多样性保护的重点 区域(Burr等, 2018), 以及分析城市绿化中的社会不平 等现象(Li和Ratti，2018)等. 在对街景照片的利用中一 个新的动向值得更多的关注, 即使用街景照片来推导 精细尺度的人类活动, 如Zhang等(2019)使用深度学习 方法分析街景照片, 估计了在城市路网上使用出租车 出行每小时的变化. 这方面的研究进一步挖掘了街景 照片作为连接城市物理空间和社会空间的桥梁的 潜力.

\section{3 量化城市生物多样性的现状和趋势}

在城市生物多样性研究中, 大数据扮演着越来越 重要的角色. 尤其是公众科学数据的应用为认识城市
生物多样性的格局, 揭示其驱动因素和机制创造了前 所未有的条件. 城市生物多样性研究和保护的发展在 很长时间内都受制于数据的可得性. 这主要是因为在 高度异质性的城市环境中进行大规模的生物多样性调 查需要相当可观的物力和人力. 同时, 由于在欧美等城 市中私人拥有大部分的城市土地, 在这些土地上进行 科学调查常常会遇到阻力. 但是, 城市地区拥有的大 量人口为公众科学的开展创造了条件, 公众科学家的 参与让调查私有土地变得相对容易(Kress，2019). 公 众科学数据具有覆盖面广、低成本的特点, 是对通过 传统的调查方法获得的城市生态数据很好的补充. 公 共科学数据已经被用于城市中鸟类(Bradsworth等, 2017; Callaghan等, 2019; Greig等, 2017)、蝴蝶(Prudic 等, 2018)、两栖动物和爬行动物(Spear等, 2017)、犬 科动物(Mueller等, 2019)、蝙蝠(Border等, 2017)、刺 猬(Williams等, 2015)和植物(Roman等, 2017)等生物的 种类多样性格局、种群动态, 以及管理和保护策略的 研究中. 与此同时, 研究者对公众数据的应用也从简 单地用其来发现格局发展到将公众数据和生态模型结 合，获得新的认识. 如Sun等(2019)发展了空间显性的 综合模型, 综合应用公众科学数据和系统调查数据, 提 高了对黑熊的丰度、成活率和补充率估计的准确度和 精度. LV等(2019)使用公众观鸟数据, 结合了占域模型, 确定了北京市重要的生态保护区域.

机会数据也日益成为城市生物多样性研究的一个 重要数据来源. 机会数据和传统的调查数据, 以及有组 织的公众科学数据不同的是, 数据并不是针对某一研 究对象而特意收集的, 往往是在为其他目的收集的数 据中包含的研究对象的可用数据. 机会数据最常见的 来源是Twitter、Flickr、Youtube等社交媒体数据. 这 类数据因为能够提供影像的地理位置、时间信息, 以 及研究对象存在的直接证据而越来越多地被用于研究 城市中的生物. 如Jagiello等(2019)使用Youtube用户上 传的录像对红松鼠和灰松鼠在欧洲的城市和森林中的 行为进行了对比分析. 机会数据另一个重要来源是政 府或私人机构在日常运行中积累的生态环境或人文环 境的监测数据. 例如, Khavaninzadeh等(2014)使用安全 监控录像分析了城市中的椴树树叶受损害的情况, 来 作为衡量城市栖息地质量的一个指标. Van Doren等 (2017)使用气象部门收集的天气雷达数据, 分析了纽 约城市灯光对迁徙鸟类的影响. 


\section{4 量化城市生态系统服务}

城市生态系统服务是指由城市内部和周边的生态 系统产生的，为城市居民所使用的生态产品或生态过 程. 由于城市生态系统服务具有自然和社会的双重属 性，且空间和时间上在生产者和使用者之间流动， 对其进行量化非常困难. 大数据在评估居民如何使用 和响应城市中的自然系统方面具有的优势为城市生态 系统服务研究创造了新的机会, 也使得这方面的研究 成为了城市生态学研究中使用大数据方法最多的 一类.

城市绿地，包括公园、保护地等自然和半自然绿 地为城市居民提供的调节、维持和文化服务对于增进 居民的健康福祉至关重要. 在传统的研究中, 研究者通 常是根据城市绿地的空间位置、大小，以及植物配置 等绿地的生物物理特性(Huang等，2017)，或者是通过 对使用者的访问调查来推测城市绿地提供的生态系统 服务(Ko和Son, 2018). 但根据绿地的生物物理特性只 能获得对绿地能提供的潜在生态系统服务的估计，而 不是真实被城市居民所使用了的生态系统服务. 而通 过对居民访问调查来对城市生态系统服务进行估计常 常由于调查样本量小、受访群体偏差等问题, 得到的 结果存在着较大的不确定性. 相比之下，使用社会感 知大数据能提供人们为什么、什么时候和如何使用城 市绿地的信息(Ilieva和McPhearson, 2018). 如基于手机 信令、地图查询和WiFi热点接入数据等大数据, 对绿 地使用人群活动进行描述或建模，能够获得对绿地使 用状况的真实描述. 在此基础上，结合对使用人群画 像、其他城市基础设施数据和社会经济数据，可以分 析影响绿地提供生态系统服务的主要因素, 并提出相 应的城市规划和设计措施来增强绿地的生态系统 服务.

目前最常见的方法是将使用者的空间轨迹和绿地 的位置及周边环境特征进行关联分析，获得对城市绿 地提供的文化服务的认识. 如Hamstead等(2018)使用 Flickr和Twitter数据分析了纽约 2143 个城市公园的使 用情况，发现了公园使用和公共交通的位置、设施、 绿地比例、周边的居民构成的相关性，以及居民在公 园访问上的不平等现象. Chen等(2018)使用每小时腾 讯微信热力图分析了深圳686个公园的使用情况，发
现在发展良好的区域使用者密度更高，公园的特性和 周边的景观特征和公园的使用是显著相关的. 手机轨 迹数据与社交媒体数据相比, 能更好地描绘个人尺度 上的行为，因此对其的应用也日益增加. 如在新加坡， 手机轨迹数据被用于计算城市保护区和和城市公园的 游㓤价值. 与传统的问卷调查相比, 手机轨迹数据可以 避免随机性错误, 旅游者的记忆带来的误差, 且成本低 (Jaung和Carrasco, 2020). 手机定位数据与高分辨率的 遥感数据被用于分析中国主要城市的居民的绿地暴露 程度动态，揭示了城市新旧区域间以及城市间居民绿 地暴露程度随时间发生的显著变化. 与传统上使用绿 地覆盖率和人均绿地面积来估算的方法相比，该方法 结合了人群分布动态和绿地的地理位置, 因此对于人 群的绿地暴露程度的估计更为合理(Song等, 2020, 2018).

除了关联使用者的空间轨迹和绿地位置外，也有 研究试图直接分析使用者感知到的生态系统服务. 如 在徐州的一项研究使用了微博上对 19 个城市公园的 7257 条评论数据，对其进行语义分析，获得了使用者 对公园提供的美观、娱乐、运动、感召、教育、文 化遗产和精神满足等文化服务的感知情况，发现美观 和娱乐是两种最常被使用者感知的服务( Dai 等, 2019). 但另一项研究发现微博登录数据和城市公园 的使用没有显著的相关性, 微博登录数据可能受到年 龄、性别和社会阶级偏见的影响(Lyu和Zhang, 2019). 因此，通过分析社交媒体内容来直接获得使用者对服 务的感知，从而判断服务的使用情况可能会存在一定 的误差.

相比于对文化服务的分析，使用大数据来分析城 市生态系统提供的调节和维持服务方面的研究相对缺 乏. 在一项研究中研究者使用POI数据, 结合地表温度 数据、地表覆盖数据, 以及建筑物体积数据, 分析了城 市地表温度和生态基础设施、人为活动的关系，发现 对地表温度影响程度依次为生态基础设施、建筑物体 积和POI密度(奕夏丽等, 2018). Burr等(2018)用街景照 片分析了城市花园在维持授粉昆虫种群的服务. Huang等(2019)采用高分辨率卫星图像数据和深度学 习的方法, 量化了城市土地利用的变化及相应的 22 种 生态系统服务的供应和需求的变化. 这些为数不多的 研究显示了大数据在这方面研究上所具有的潜力亟待 开发. 


\section{4 城市生态学中应用大数据所面临的挑战}

\section{1 大数据与城市生态学研究融合的问题}

生态学已经进入了大数据时代, 如何将基于过程 的传统生态学研究和基于大数据驱动的生态学研究有 机整合既是重大挑战，也是机遇(于贵瑞等，2018). 作 为生态学的一个分支, 城市生态学同样也面临着这个 挑战和机遇. 对于大数据在科学研究中的作用一直存 在着不同的认识. 一些学者认为大数据将取代传统的 科学研究范式. Anderson(2008)发表的著名宣言

“数据洪流让科学方法变得过时”是这类观点的代 表. 而另外一些学者则认为诸如大数据将取代传统数 据，而不是作为其补充的观点是“大数据浮夸”(Kress, 2019). 缺乏对大数据作用的明确定位, 以及如何和传 统的研究模式结合的思路, 是当前众多的生态学家在 研究中并不接受和使用大数据的主要原因(Peters等, 2014).

大数据科学提倡不预设假设, 从海量的数据中去 发现规律, 注重相关性而不是因果关系. 这种模式与 传统的科学研究所遵循的“提出科学假设-收集数据证实或推翻假设”的模式截然不同. 不可否认的是，在 先验知识不足以描述生态过程的时候，通过数据挖掘 技术分析大数据, 在一定程度上能够识别数据中多元 的格局和重要的驱动因子(Hochachka等，2007). 但是 依赖于大数据去发现城市生态学中的规律会遇到种种 问题, 包括: 大数据发现的规律是否有科学意义? 一些 重要但少量发生的现象能否被捕捉到? 能否基于数据 相关性来进行精准的预测? (Rabari和Storper, 2015). 于 贵瑞等(2018)非常明确地指出, 由于生态系统组成的 多样性及其相关关系的复杂性，不管获得的数据量有 多大，如果没有大理论支撑，大数据将会在很大程度 上失去有效性.

但是, 如果因为这些问题而在城市生态研究中放 弃使用大数据，在一定程度上等同于放弃了城市生态 学的未来. LaDeau等(2017)预言在生态学研究中, 那些 能够获得大数据, 能够综合使用数据去产生新的假设 和模型的生态学家将处于一个有利位置. 他们能够在 需要的尺度上推导生态系统的功能, 并能够产生对管 理有用的预测，从而增加生态系统的稳定性. 这些预 言对于城市生态学研究来说尤其相关. 陈发虎等 (2019)指出大数据作为现代化的研究手段, 有助于解
决学科交融中的突出科学问题. 城市生态学具有的自 然科学和社会科学高度交叉的学科属性, 城市作为一 个巨大的数据工厂的现实, 以及在前面所讨论的应用 大数据对城市生态研究的种种促进之处, 都决定了大 数据在城市生态学研究中可以发挥独特而重要的 作用.

阻碍大数据科学和传统的城市生态学深入融合的 原因既来自大数据本身, 也来自城市生态学研究模式. 大数据的信噪比低、来源多样(Wang和Moriarty, 2018), 数据常受到选择性偏差的影响(Scott, 2018), 所 以使用前需要整合. 大数据常是能找到的数据或便利 样本, 可能并不能很好的代表总体(Franke等，2016). 此外, 大数据通常捕捉被公开表达的数据, 包括输入 的信息、刷卡、扫描、人们的行动和行为数据、移动 的物体轨迹等, 但在捕捉人们复杂的情绪、价值、信 念和观点等信息时存在不足(Kitchin，2013). 另一方 面, 传统的科学研究追求理解现象底层的过程, 视其 为发现知识和解决问题的过程. 所以基于对大量质量 参差不齐的数据所做的相关性分析对这个过程来说是 无用的(Peters等, 2014). 此外, 一些传统的生态数据分 析方法无法处理大而复杂的数据集, 例如很多大数据 是扁平化的, 即变量的数目多于观测的数量, 这对传 统的统计分析方法构成了挑战(Franke等, 2016).

要在城市生态学研究中使用好大数据, 首先必须 将大数据的分析建立在对生态系统的过程、格局和模 式深入的理解发展出来的知识和直觉之上(Peters和 Okin, 2017; 于贵瑞等, 2018). 其次, 需要发展能够促进 在城市生态学研究中应用大数据的方法. 例如, 发展开 放式的数据分析方法, 能够进行迭代学习, 使得数据分 析能伴随着数据类型、数量和增长率的增加而提高效 率(Peters等，2014). 发展在生态学研究中应用人工智 能的方法. 如基于神经网络对生态系统离散状态和连 续系统行为进行预测方法, 基于卷积神经网络的遥 感、无人机和监控图像的分析方法，基于混合进化算 法的阈值分析和元分析方法等(Recknagel和Staiano, 2019). 发展能够整合大数据与经验或理论模型结合的 方法，例如英国一项研究发展了图像识别结合背景知 识的方法, 让机器像专家一样思考, 将仅用图像识别 时获得的 $48.2 \%$ 的识别准确度提高了 $9.1 \%$ (Terry等, 2020). 此外, 发展将大数据和实验数据混合使用的方 法. 如在使用大数据进行时间序列分析时加入实验数 
据能够降低时间序列的变异程度, 提高模型的分析能 力, 并增强因果关系的信号(Benedetti-Cecchi等, 2018). 最后, 发展基于过去经验的自动机器学习方法, 来指引 个人进行假设检验或修正之前提出的假设(Peters等, 2014). 根据城市生态学研究的核心问题和特点, 结合 这些途径, 可以提出城市生态学和大数据方法融合的 框架(图1).

\section{2 大数据应用面临的数据获取问题}

城市生态研究中应用大数据的一个主要的限制性 因素是数据获取问题. 城市生态学研究目前使用的大 数据的可获取度差异很大(图2). 迄今为止，城市生态 研究中使用的大数据大多是可公开获取的开源数据, 如源自社交媒体的数据，或使用网络爬虫方式从网页 上抓取的数据. 而质量更好的、能够精确地描绘城市 居民的个体行为的大数据常常是掌握在移动服务供应 商或大型网络公司手中(Kitchin, 2013). 基于数据具有 的商业价值, 这些公司缺乏与研究者合作将数据用于 城市生态研究的动力. 在为数不多的合作案例中, 商 业公司与研究者共享的数据量非常有限. 如Twitter可 以免费提供 14 天的数据, 研究者需要更多的历史数据 需要向商业公司购买．以至于有研究者指出，当前大 数据主要的用途是商业用途，而不是用于解决社会面 临的问题(Shin和Choi，2015). 此外，政府和民众对于 大数据隐私和安全性的顾虑日益增加，欧盟颁布了 《通用数据保护条例》，中国也正式实施了《信息安 全技术个人信息安全规范》，这些法规提高了获取城 市大数据的难度. 通过大数据、人工智能技术与区块 链技术的结合来提高大数据分析的安全性的尝试(A1lam和Dhunny, 2019)可能会提供技术上的解决方案, 但 解决因安全顾虑而导致的大数据获取困难还需要制度 上的解决办法.

城市大数据中迄今为止可获得性最差、利用程度 最低的一类数据是政府部门拥有的数据. 政府部门在 运行过程中积累了大量的数据. 例如, 重庆的 51 个政 府部门中平均每个部门有 5 6 个数据系统，整个政府 有近300个系统在运行 (邓晖，2016). 这些数据涉及城 市生态系统的方方面面，如园林和林业部门调查的动 植物种类和丰富度、栖息地状态，环境部门调查的空 气、水和土壤状态等. 这类数据的更新频率较基于网 络或物联网获取的大数据慢，一般时间尺度在日到数

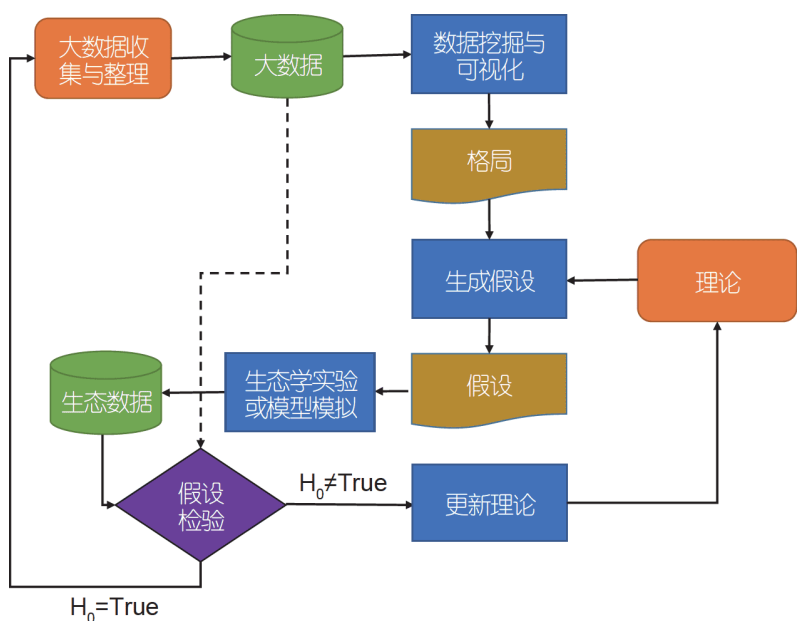

图 1 城市生态学研究与大数据融合的框架示意图 虚线表示大数据可以和生态数据混合进行假设检验，也可以仅使用 生态数据进行假设检验

年之间. 但在各城市纷纷发展基于物联网的生态环境 监测体系的新形势下，一些生态环境数据已经能够做 到实时获取(金颖和于㢷，2018). 因此，推进政府管理 的生态大数据的开放，包括依托环境保护部门的政府 网站建立生态数据开放平台(高渝斐, 2018), 制定数据 标准，提高数据的规范性等举措(金颖和于㢷，2018), 将会极大地推动政府拥有的数据在城市生态研究中的 应用. 遗憾的是，虽然政府拥有的数据具有很高的价 值，但往往出于安全性，以及数据处理和共享所需的 资源的考虑，政府部门开放数据的意愿低(邓晖, 2016), 迫切需要自上而下的推动共享和开放.

城市生态学者本身对数据分享所持的态度也在很 大程度上影响了城市生态研究相关的大数据的可得 性. 生态学研究往往规模小. 受资源限制, 研究者个人 数据保存和分享的能力有限, 且缺乏分享数据的动力, 造成了大量数据虽然具有科研价值，但不为人知的现 象. 如果城市生态学研究者产生的数据要有大的影响, 必须注意数据的可被发现性、可用性和科学上的严谨 性, 并提供记录的元数据和采用开放文档的形式, 便于 数据在不同的平台之间分享(Kress, 2019). 这不但需要 提高数据的规范化和构建公共数据分享平台(Hampton等, 2013), 还需要形成开放共享、协同合作的大数 据文化. 一些如数据众包采集、数据出版、数据标识 等新的数据获取和知识产权保护方式的深入应用会在 一定程度上促进生态数据的分享(于贵瑞等, 2018). 此 


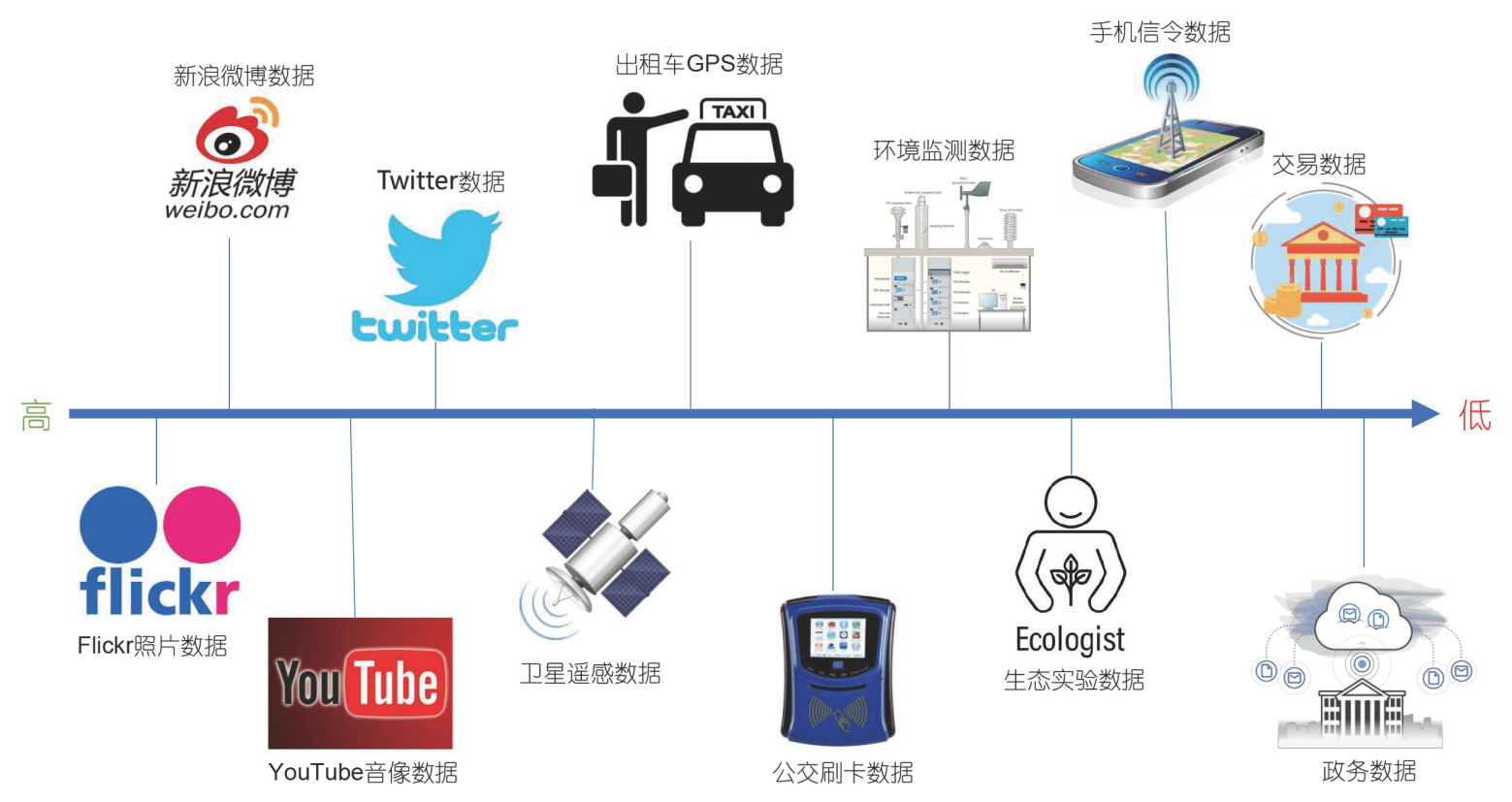

图 2 城市生态研究中大数据的可获得性

外, 还需要发展在分析中整合大数据和城市生态学者 收集的小数据的方法, 降低数据的整合过程中不确定 性扩增和数据的偏差, 增加使用大数据分析的可重现 性和效率.

\section{3 大数据应用面临的技术门槛问题}

城市生态学研究者如果想在研究中使用大数据, 必需要克服一些技术上的门槛. 这些门槛存在于大数 据的储存、检索、分析和可视化等多个方面. 首先是 大量数据如何储藏、检索和分析的问题. 很多传统的 统计分析方法需要使用所有的数据进行分析, 无法处 理分布式的数据, 但大数据的分布式储存往往是用于 解决大数据所具有的巨大数据量的主要方法(Franke 等, 2016). 此外, 模型模拟是城市生态学研究的重要工 具, 或作为一些在城市环境中无法进行的控制性实验 的替代, 或对未来的情况进行预测. 但是, 有研究发现 模型模拟算法的迭代性特点和典型的大数据模式不能 很好地吻合, 需要花费大量的时间和算力反复调整算 法和模拟(Caíno-Lores等, 2018). 大数据科学家们对这 些问题提出了一些解决方案, 包括发展在数据收集或 生成的各个环节减少数据的量, 去掉圥余的数据的同 时保持最小程度的信息损失的方法, 发展结合并行编 程计算和基于大数据的数据抽象混合方法的工作流,
以及提高运算内存的能力等(Ang和Seng, 2016; CaínoLores等, 2018). 但很明显, 这些解决方案对数据分析 能力提出了很高的要求, 难以期望城市生态学研究人 员能够独立实现. 即使是一些数据科学家认为是简单 的技术, 例如使用深度学习模型来挖掘大量图像中的 信息, 对城市生态学研究人员也可能是一种挑战. 因 为当前的生态学在数据分析方面的教育主要以传统的 统计分析方法为主, 很少涉及大数据分析技术的培训.

大数据的可视化是城市生态学者面临的另一个技 术门槛. 数据的可视化是获得可以行动的深层认识的 关键(Scott，2018). 当前大数据的可视化受限于技术, 往往需要在可视化的效力和直观性之间达成妥协. 妥 协的后果是一次只能展示部分的数据, 导致视觉上的 偏差(Franke等, 2016). 更为严重的是, 如果可视化是 基于不透明的、以美观为主的算法生成的, 使用者将 无法判断结果的准确程度和显著性(Ekbia等, 2015). 针对这些问题目前有研究人员提出了一些解决方案, 如通过随机矩阵映射来压缩数据, 找到最优的代表数 据等(Franke等, 2016). 但和前一问题相似的是, 城市 生态研究人员很难独自去应用这些解决方案, 常常是 依赖现成的工具来解决可视化的需求, 因而很可能会 无法意识到工具中存在的不足, 造成认识上的偏差.

支撑大数据分析技术所需要的软硬件设备也可能 
成为在城市生态研究中应用大数据方法的门槛. 大数 据的储存、检索和计算需要良好的数据处理基础设施 和强大的数据管理操作能力, 但城市生态学研究人员 很难具备这些设施和能力, 这在一些欠发达国家和地 区尤其突出. 除了和数据科学家合作来解决软硬件的 需求之外, 云计算的应用也是一个可能的解决方案. 例如Google公司提供的Google Earth Engine遥感影像 处理云平台就集成了卫星遥感大数据获取、存储、分 析和可视化的功能, 在推动全球对卫星遥感数据的分 析和应用方面发挥了巨大的作用(Gorelick等，2017). 但要在城市生态研究中发展和使用类似的云计算平 台，很大程度上将取决于提供云计算服务的供应商是 否愿意开放平台或降低价格，让一般城市生态研究人 员也能够使用.

\section{5 未来城市生态学研究中大数据应用的重 点方向}

如何增加城市的可持续性将是城市生态学未来十 年的核心研究问题, 在回答这个问题的同时, 城市生态 学将完成向“为了城市的生态学”的研究范式的转变. 城市生物多样性、城市生态系统服务和人类健康福 祉、城市生态系统过程的监测, 以及城市生态研究和 城市规划和管理的结合将是城市生态学未来研究中关 注的重点. 大数据方法在这些方向上都具有良好的应 用前景

\section{1 城市生物多样研究}

大数据应用已经成为了国际生物多样性研究的前 沿内容(张健, 2017). 在城市生物多样性研究中, 大数 据在生物多样性的监测和保护上有很大的发展空间, 未来可以重点关注以下的方向: (1) 城市生物多样性全 覆盖监测方法. 基于遥感、视频和传感器网络等技术 手段, 建立起对城市生物及其栖息环境进行连续观测 的监测网络, 其中遥感解决监测覆盖的空间范围问题, 而视频、传感器网络等提供细节内容. (2) 大量监测数 据的分析方法. 如发展图像识别和声音识别等技术, 让 识别的精度达到或超越专家人工识别的水平. 深度学 习在该方向上具有极大的应用潜力(郭庆华等，2020). (3) 发展公众科学方法. 包括开发移动平台应用和其 他网络数据收集工具, 并通过游戏和在线教育等方式
来增加公众的参与; 促进公众科学数据收集的标准化, 推动在研究中对公众数据的使用. (4) 发现和利用机会 数据的新方法. 包括利用安全监控录像进行城市植物 的物候变化、城市家养和野生动物活动的研究, 或利 用城市噪声监测数据对城市鸟类的种群动态进行监测 等. 这类数据在生物多样性研究方面具有很大潜力, 但 目前对其应用较少.

\section{2 城市生态系统服务与健康福祉}

城市生态系统服务是连接城市中自然系统和社会 系统的桥梁. 大数据所具备的刻画人的行为和社会网 络的能力为研究城市生态系统服务创造了前所未有机 会. 未来值得注意的研究方向包括: (1) 生态系统服务 定量分析方法. 发展综合社会经济大数据、地理空间 大数据、遥感数据和生态系统模型, 对生态系统服务 的产生和流动进行量化和预测的方法，尤其需要关注 调节和供应服务的定量化分析. (2) 生态系统服务产 生的价值和福祉的估算方法. 使用地理空间大数据、 环境监测大数据、社会经济数据、健康医疗数据等多 源数据, 结合归因模型, 估算生态系统服务产生的价值 和健康福祉. (3) 生态系统服务的感知. 发展大数据的 纠偏方法和情感分析方法, 基于社交媒体数据, 分析居 民对城市生态系统服务的认知和评价.

\section{3 城市生态系统过程的监测}

在城市生态研究中常常因为受制于数据的连续性 和覆盖范围, 而缺乏对系统过程及其动态的考虑. 大数 据方法可以在一定程度上弥补这个不足，值得关注的 方向有：（1）基于物联网技术的城市生态系统监测体 系. 包括获取和集成各种传感器生成多尺度、多源的 监测数据, 并基于云计算、深度学习等分析技术, 实 现对城市生态系统过程的实时、连续监测. 在该方向 上可以借鉴基于物联网和智能装置的自然生态系统监 测网络的成功经验( $\mathrm{Li}$ 等, 2019). (2) 基于社会经济大数 据的城市生态系统物质流和能流分析．如基于商品流 动数据和载货卡车的全球定位数据进行城市次生物质 来源的分析(Yeow和Cheah, 2019). (3) 基于政府公共服 务数据的物质流和能流分析. 如基于智能电表、水 表、燃气表和环境传感器监测数据等分析城市生态系 统中能源和水的消耗，以及产生的废水、废气和废热 的流动和其生态效应. 


\section{4 城市生态研究与城市规划和管理的结合}

城市生态学未来的发展在很大程度上将取决于 能否回答城市在可持续发展中遇到的问题，以及如 何和城市规划和管理更好地结合. 大数据的应用为 增加城市生态研究与城市规划和管理的结合提供了 新的机会. 未来值得关注的方向包括：(1) 发展从整 体上认识城市数据的方法. 城市规划中常需要对一 些复杂城市问题的整体认识，如能够重复使用、可 整合异质性的数据源的轻量级语义模型(Cormenza$\mathrm{na}$ 等，2018)等工具的开发将有助于实现这个目标. (2) 研发基于地理空间大数据和遥感数据, 能够更好 地表征人类活动和生态物理过程的虚拟模型，对各 种情境下城市规划或管理决策可能的影响进行更为 真实的模拟. (3) 发展基于城市大数据来快速和准确 地诊断城市面临的问题，并提出优化的规划和管理 对策的能力.

\section{6 结语}

McDonald(2016)指出: 当前我们所处的城市世纪 是自工业革命以来最重要的人类变革. 作为城市生态 学家能够去观察、学习甚至去影响这场革变革是一 件非常幸运的事. 城市生态学研究在未来将进一步聚 焦城市生态系统和人类社会之间的复杂相互作用, 为 实现城市的可持续发展提供可行的解决方案. 实现这 个目标需要城市生态学研究人员在新的理论、方法 支撑下，获得对城市生态系统的深入认识. 城市在大 数据生产中的核心地位使得大数据科学和城市生态 学的结合已成为一个必然的趋势. 但目前在城市生态 研究中大数据的应用依然有限, 已有的应用也大多是 停留在对大数据的潜力浅层次挖掘的层面. Alberti (2017)对大数据在城市科学中的应用状况所做的判 断在当前依然成立，即大数据分析只是回答了能够被 描述的问题, 真正的挑战是将这些前所未有的数据转 化成对城市系统的新认识. 大数据在回答城市生态学 未来十年的核心研究问题, 推动城市生态学研究范式 的转变中具有重要的潜力. 城市生态学研究人员亟需 充分挖掘这个潜力, 将大数据从一个吸引眼球的概念 转化为能够指导城市规划和决策管理的城市生态学 研究成果.

\section{参考文献}

陈发虎, 傅伯杰, 夏军, 吴铎, 吴绍洪, 张镱锂, 孙航, 刘禹, 方小敏, 秦 伯强, 李新, 张廷军, 刘宝元, 董治宝, 侯书贵, 田立德, 徐柏青, 董 广辉, 郑景云, 杨威, 王金金, 李再军, 王飞, 胡振波, 王杰, 刘建宝, 陈 建徽, 黄伟, 侯居峙, 蔡秋芳, 隆浩, 姜明, 胡亚鲜, 冯晓明, 莫兴国, 杨晓燕, 张东菊, 王秀红, 尹云鹤, 刘晓晨. 2019. 近70年来中国自 然地理与生存环境基础研究的重要进展与展望. 中国科学 $\mathrm{D}$ 辑: 地球科学, 49: 1659-1696

邓晖. 2016. 城市大数据的生态模型及应用. 大数据, (2): 68-75

高渝斐. 2018. 我国城市生态系统领域相关技术政策初探. 城市建筑, (7): 73-77

郭庆华, 金时超, 李敏, 杨秋丽, 徐可心, 巨袁臻, 张菁, 宣晶, 刘瑾, 苏 艳军, 许强, 刘瑜. 2020. 深度学习在生态资源研究领域的应用: 理论、方法和挑战. 中国科学: 地球科学, 50: 1354-1373

金颖, 于㢷. 2018. 物联网技术在城市生态保护和修复中的应用策略. 城市建筑, (17): 108-111

刘瑜, 詹朝晖, 朱递, 柴彦威, 马修军, 乌伦. 2018. 集成多源地理大数 据感知城市空间分异格局. 武汉大学学报(信息科学版), 43: 327335

栾夏丽, 韦胜, 韩善锐, 李小婷, 杨文宇, 刘茂松, 徐驰. 2018. 基于城 市大数据的热场格局形成机制及主导因素的多尺度研究. 应用 生态学报, 29: 2861-2868

于贵瑞, 何洪林, 周玉科. 2018. 大数据背景下的生态系统观测与研 究. 中国科学院院刊, 33: 832-837

马世俊, 王如松. 1984. 社会-经济-自然复合生态系统. 生态学报, 4: $1-8$

张健. 2017. 大数据时代的生物多样性科学与宏生态学. 生物多样性, 25: $355-363$

Alberti M. 2017. Grand challenges in urban science. Front Built Environ, 3: 6

Allam Z, Dhunny Z A. 2019. On big data, artificial intelligence and smart cities. Cities, 89: 80-91

Anderson C. 2008. The end of theory: The data deluge makes the scientific method obsolete. Wired, 23 June 2008

Ang L M, Seng K P. 2016. Big sensor data applications in urban environments. Big Data Res, 4: 1-12

Barot S, Abbadie L, Auclerc A, Barthélémy C, Bérille E, Billet P, Clergeau P, Consales J N, Deschamp-Cottin M, David A, Devigne C, Dham V, Dusza Y, Gaillard A, Gonzalez E, Hédont M, Labarraque D, Le Bastard A M, Morel J L, Petit-Berghem Y, Rémy E, Rochelle-Newall E, Veyrières M. 2019. Urban ecology, stakeholders and the future of ecology. Sci Total Environ, 667: 475-484

Benedetti-Cecchi L, Bulleri F, Dal Bello M, Maggi E, Ravaglioli C, 
Rindi L. 2018. Hybrid datasets: Integrating observations with experiments in the era of macroecology and big data. Ecology, 99: 2654-2666

Boeing G. 2019. Spatial information and the legibility of urban form: Big data in urban morphology. J Inf Manag, https://doi.org/10.1016/ j.ijinfomgt.2019.09.009

Border J A, Newson S E, White D C J, Gillings S. 2017. Predicting the likely impact of urbanisation on bat populations using citizen science data, a case study for Norfolk, UK. Landscape Urban Plan, 162: $44-55$

Bradsworth N, White J G, Isaac B, Cooke R. 2017. Species distribution models derived from citizen science data predict the fine scale movements of owls in an urbanizing landscape. Biol Conserv, 213: $27-35$

Burr A, Schaeg N, Hall D M. 2018. Assessing residential front yards using google street view and geospatial video: A virtual survey approach for urban pollinator conservation. Appl Geogr, 92: 12-20

Cai B, Li X, Seiferling I, Ratti C. 2020. Treepedia 2.0: Applying deep learning for large-scale quantification of urban tree cover. In: 2018 IEEE International Congress on Big Data. Seattle. 49-56

Cai J, Huang B, Song Y. 2017. Using multi-source geospatial big data to identify the structure of polycentric cities. Remote Sens Environ, 202: $210-221$

Caíno-Lores S, Lapin A, Carretero J, Kropf P. 2020. Applying big data paradigms to a large scale scientific workflow: Lessons learned and future directions. Future Generation Comput Syst, 110: 440-452

Callaghan C T, Bino G, Major R E, Martin J M, Lyons M B, Kingsford R T. 2019. Heterogeneous urban green areas are bird diversity hotspots: Insights using continental-scale citizen science data. Landscape Ecol, 34: 1231-1246

Cao R, Tu W, Yang C, Li Q, Liu J, Zhu J, Zhang Q, Li Q, Qiu G. 2020. Deep learning-based remote and social sensing data fusion for urban region function recognition. ISPRS J Photogrammetry Remote Sens, 163: 82-97

Chen Y, Liu X, Gao W, Wang R Y, Li Y, Tu W. 2018. Emerging social media data on measuring urban park use. Urban Forry Urban Green, 31: $130-141$

Childers D, Cadenasso M, Grove J, Marshall V, McGrath B, Pickett S 2015. An ecology for cities: A transformational nexus of design and ecology to advance climate change resilience and urban sustainability. Sustainability, 7: 3774-3791

Cormenzana B, Fabregas F, Marinescu M C, Marrero M. 2018. An ontology for ecological urbanism: SUM+Ecology. In: AAAI-2014 Semantic Cities. Semantic Cities: Beyond Open Data to Models, Standards and Reasoning. 2-8

Dai P, Zhang S, Chen Z, Gong Y, Hou H. 2019. Perceptions of cultural ecosystem services in urban parks based on social network data. Sustainability, 11: 5386

Durden J M, Luo J Y, Alexander H, Flanagan A M, Grossmann L. 2017. Integrating "Big Data" into aquatic ecology: Challenges and opportunities. Limnol Oceanogr Bull, 26: 101-108

Economist. 2012. The New Local. Economist, October 27, 14

Ekbia H, Mattioli M, Kouper I, Arave G, Ghazinejad A, Bowman T, Suri V R, Tsou A, Weingart S, Sugimoto C R. 2015. Big data, bigger dilemmas: A critical review. J Assn Inf Sci Tec, 66: 1523-1545

Franke B, Plante J F, Roscher R, Lee E A, Smyth C, Hatefi A, Chen F, Gil E, Schwing A, Selvitella A, Hoffman M M, Grosse R, Hendricks D, Reid N. 2016. Statistical inference, learning and models in big data. Int Stat Rev, 84: 371-389

Frantzeskaki N, McPhearson T, Collier M J, Kendal D, Bulkeley H, Dumitru A, Walsh C, Noble K, van Wyk E, Ordóñez C, Oke C, Pintér L. 2019. Nature-based solutions for urban climate change adaptation: Linking science, policy, and practice communities for evidence-based decision-making. Bioscience, 69: 455-466

Gandy M. 2015. From urban ecology to ecological urbanism: An ambiguous trajectory. Area, 47: 150-154

Gong J, Liu C, Huang X. 2020. Advances in urban information extraction from high-resolution remote sensing imagery. Sci China Earth Sci, 63: 463-475

Gong P, Chen B, Li X, Liu H, Wang J, Bai Y, Chen J, Chen X, Fang L, Feng S, Feng Y, Gong Y, Gu H, Huang H, Huang X, Jiao H, Kang Y, Lei G, Li A, Li X, Li X, Li Y, Li Z, Li Z, Liu C, Liu C, Liu M, Liu S, Mao W, Miao C, Ni H, Pan Q, Qi S, Ren Z, Shan Z, Shen S, Shi M, Song Y, Su M, Ping Suen H, Sun B, Sun F, Sun J, Sun L, Sun W, Tian T, Tong X, Tseng Y, Tu Y, Wang H, Wang L, Wang X, Wang Z, Wu T, Xie Y, Yang J, Yang J, Yuan M, Yue W, Zeng H, Zhang K, Zhang N, Zhang T, Zhang Y, Zhao F, Zheng Y, Zhou Q, Clinton N, Zhu Z, Xu B. 2020. Mapping essential urban land use categories in China (EULUC-China): Preliminary results for 2018. Sci Bull, 65: 182-187

Gorelick N, Hancher M, Dixon M, Ilyushchenko S, Thau D, Moore R. 2017. Google Earth Engine: Planetary-scale geospatial analysis for everyone. Remote Sens Environ, 202: 18-27

Greig E I, Wood E M, Bonter D N. 2017. Winter range expansion of a hummingbird is associated with urbanization and supplementary feeding. Proc R Soc B, 284: 20170256

Grimm N B, Faeth S H, Golubiewski N E, Redman C L, Wu J, Bai X, Briggs J M. 2008. Global change and the ecology of cities. Science, 319: 756-760

Hampton S E, Strasser C A, Tewksbury J J, Gram W K, Budden A E, Batcheller A L, Duke C S, Porter J H. 2013. Big data and the future of ecology. Front Ecol Environ, 11: 156-162 
Hamstead Z A, Fisher D, Ilieva R T, Wood S A, McPhearson T, Kremer P. 2018. Geolocated social media as a rapid indicator of park visitation and equitable park access. Comput Environ Urban Syst, 72: $38-50$

Helbich M, Yao Y, Liu Y, Zhang J, Liu P, Wang R. 2019. Using deep learning to examine street view green and blue spaces and their associations with geriatric depression in Beijing, China. Environ Int, 126: $107-117$

Hochachka W M, Caruana R, Fink D, Munson A, Riedewald M, Sorokina D, Kelling S. 2007. Data-mining discovery of pattern and process in ecological systems. J Wildl Manage, 71: 2427-2437

Hu T, Yang J, Li X, Gong P. 2016. Mapping urban land use by using landsat images and open social data. Remote Sens, 8: 151

Huang C, Yang J, Lu H, Huang H, Yu L. 2017. Green spaces as an indicator of urban health: Evaluating its changes in 28 mega-cities. Remote Sens, 9: 1266

Huang X, Han X, Ma S, Lin T, Gong J. 2019. Monitoring ecosystem service change in the City of Shenzhen by the use of high-resolution remotely sensed imagery and deep learning. Land Degrad Dev, 30: $1490-1501$

Huang X, Wang Y, Li J, Chang X, Cao Y, Xie J, Gong J. 2020. Highresolution urban land-cover mapping and landscape analysis of the 42 major cities in China using ZY-3 satellite images. Sci Bull, 65: 1039-1048

International Energy Agency, IEA. 2016. Energy Technology Perspectives 2016. Paris: International Energy Agency

Ilieva R T, McPhearson T. 2018. Social-media data for urban sustainability. Nat Sustain, 1: 553-565

Jagiello Z A, Dyderski M K, Dylewski Ł. 2019. What can we learn about the behaviour of red and grey squirrels from YouTube? Ecol Inf, 51: 52-60

Jaung W, Carrasco L R. 2020. Travel cost analysis of an urban protected area and parks in Singapore: A mobile phone data application. J Environ Manage, 261: 110238

Khavaninzadeh A R, Veroustraete F, Buytaert J A N, Samson R. 2014. Leaf injury symptoms of Tilia sp. as an indicator of urban habitat quality. Ecol Indicators, 41: 58-64

Kitchin R. 2013. Big data and human geography. Dialogues Human Geography, 3: 262-267

Ko H, Son Y. 2018. Perceptions of cultural ecosystem services in urban green spaces: A case study in Gwacheon, Republic of Korea. Ecol Indicators, 91: 299-306

Kress M M. 2019. Big data for ecological models. In: Jørgensen S E, Fath B D, eds. Encyclopedia of Ecology volume 1. Amsterdam: Eslsevier. 11-20

LaDeau S L, Han B A, Rosi-Marshall E J, Weathers K C. 2017. The next decade of big data in ecosystem science. Ecosystems, 20: 274283

Lenormand M, Ramasco J J. 2016. Towards a better understanding of cities using mobility data. Built Eviron, 42: 356-364

Li X, Ratti C. 2018. Mapping the spatial distribution of shade provision of street trees in Boston using Google Street View panoramas. Urban Forry Urban Green, 31: 109-119

Li X, Zhang C, Li W, Ricard R, Meng Q, Zhang W. 2015. Assessing street-level urban greenery using Google Street View and a modified green view index. Urban Forry Urban Green, 14: 675-685

Li X, Zhao N, Jin R, Liu S, Sun X, Wen X, Wu D, Zhou Y, Guo J, Chen S, Xu Z, Ma M, Wang T, Qu Y, Wang X, Wu F, Zhou Y. 2019. Internet of Things to network smart devices for ecosystem monitoring. Sci Bull, 64: 1234-1245

Lin T, Grimm N B. 2014. Comparative study of urban ecology development in the U.S. and China: Opportunity and challenge. Urban Ecosyst, 18: 599-611

Liu Y, Liu X, Gao S, Gong L, Kang C, Zhi Y, Chi G, Shi L. 2015. Social sensing: A new approach to understanding our socioeconomic environments. Ann Assoc Am Geogr, 105: 512-530

Lv Z, Yang J, Wielstra B, Wei J, Xu F, Si Y. 2019. Prioritizing green spaces for biodiversity conservation in Beijing based on habitat network connectivity. Sustainability, 11: 2042

Lyu F, Zhang L. 2019. Using multi-source big data to understand the factors affecting urban park use in Wuhan. Urban Forry Urban Green, 43: 126367

McDonald R I. 2016. Urban ecology for the urban century. Ecosyst Health Sustainabil, 2: e01221

McDonnell M J, Pickett S T A. 1990. Ecosystem structure and function along urban-rural gradients: An unexploited opportunity for Ecology. Ecology, 71: 1232-1237

McPhearson T, Pickett S T A, Grimm N B, Niemelä J, Alberti M, Elmqvist T, Weber C, Haase D, Breuste J, Qureshi S. 2016. Advancing urban ecology toward a science of cities. Bioscience, 66 : 198-212

Middel A, Lukasczyk J, Zakrzewski S, Arnold M, Maciejewski R. 2019. Urban form and composition of street canyons: A humancentric big data and deep learning approach. Landscape Urban Plan, 183: $122-132$

Mueller M A, Drake D, Allen M L. 2019. Using citizen science to inform urban canid management. Landscape Urban Plan, 189: 362371

Paldino S, Kondor D, Bojic I, Sobolevsky S, González M C, Ratti C. 2016. Uncovering urban temporal patterns from geo-tagged photography. PLoS One, 11: e0165753

Park R, Ernest W B, Roderick D M. 1925. The City. Chicago: 
University of Chicago Press. 239

Pataki D E. 2015. Grand challenges in urban ecology. Front Ecol Evol, 3: 57

Peters D P C, Havstad K M, Cushing J, Tweedie C, Fuentes O, Villanueva-Rosales N. 2014. Harnessing the power of big data: Infusing the scientific method with machine learning to transform ecology. Ecosphere, 5: art67

Peters D P C, Okin G S. 2017. A toolkit for ecosystem ecologists in the time of big science. Ecosystems, 20: 259-266

Pickett S T A, Cadenasso M L, Baker M E, Band L E, Boone C G, Buckley G L, Groffman P M, Grove J M, Irwin E G, Kaushal S S, LaDeau S L, Miller A J, Nilon C H, Romolini M, Rosi E J, Swan C M, Szlavecz K. 2020. Theoretical perspectives of the Baltimore ecosystem study: Conceptual evolution in a social-ecological research project. Bioscience, 70: 297-314

Prudic K L, Oliver J C, Brown B V, Long E C. 2018. Comparisons of citizen science data-gathering approaches to evaluate urban butterfly diversity. Insects, 9: 186

Rabari C, Storper M. 2015. The digital skin of cities: Urban theory and research in the age of the sensored and metered city, ubiquitous computing and big data. Camres, 8: 27-42

Recknagel F, Staiano A. 2019. Editorial: Analysis and synthesis of ecological data by machine learning. Ecol Inf, 53: 100971

Roman L A, Scharenbroch B C, Östberg J P A, Mueller L S, Henning J G, Koeser A K, Sanders J R, Betz D R, Jordan R C. 2017. Data quality in citizen science urban tree inventories. Urban Forry Urban Green, 22: 124-135

Scott E M. 2018. The role of Statistics in the era of big data: Crucial, critical and under-valued. Stat Probab Lett, 136: 20-24

Shin D H, Choi M J. 2015. Ecological views of big data: Perspectives and issues. Telematics Inf, 32: $311-320$

Shirtcliff B. 2015. Big data in the big easy: How social networks can improve the place for young people in cities. Landscape J, 34: 161176

Shochat E, Warren P, Faeth S. 2006. Future directions in urban ecology. Trends Ecol Evol, 21: 661-662

Song Y, Chen B, Kwan M P. 2020. How does urban expansion impact people's exposure to green environments? A comparative study of 290 Chinese cities. J Clean Prod, 246: 119018

Song Y, Huang B, Cai J, Chen B. 2018. Dynamic assessments of population exposure to urban greenspace using multi-source big data. Sci Total Environ, 634: 1315-1325

Sotomayor-Gómez B, Samaniego H. 2020. City limits in the age of smartphones and urban scaling. Comput Environ Urban Syst, 79: 101423

Spear D M, Pauly G B, Kaiser K. 2017. Citizen Science as a tool for augmenting museum collection data from urban areas. Front Ecol Evol, 5: 86

Sukopp H. 2008. On the early history of urban ecology in Europe. In: Marzluff J M, Shulenberger E, Endlicher W, Alberti M, Bradley G, Ryan C, Simon U, ZumBrunnen C, eds. Urban Ecology: An International Perspective on the Interaction Between Humans and Nature. Boston: Springer. 79-97

Sukopp H, Weiler S. 1988. Biotope mapping and nature conservation strategies in urban areas of the Federal Republic of Germany. Landscape Urban Plan, 15: 39-58

Sun C C, Royle J A, Fuller A K. 2019. Incorporating citizen science data in spatially explicit integrated population models. Ecology, 100: 02777

Tanner C J, Adler F R, Grimm N B, Groffman P M, Levin S A, MunshiSouth J, Pataki D E, Pavao-Zuckerman M, Wilson W G. 2014. Urban ecology: Advancing science and society. Front Ecol Environ, 12: $574-581$

Terry J C D, Roy H E, August T A, Altwegg R. 2020. Thinking like a naturalist: Enhancing computer vision of citizen science images by harnessing contextual data. Methods Ecol Evol, 11: 303-315

United Nations, Department of Economic and Social Affairs, Population Division. 2019. World Urbanization Prospects: The 2018 Revision (ST/ESA/SER.A/420). New York: United Nations. 126

Van Doren B M, Horton K G, Dokter A M, Klinck H, Elbin S B, Farnsworth A. 2017. High-intensity urban light installation dramatically alters nocturnal bird migration. Proc Natl Acad Sci USA, 114: 11175-11180

Wang S, Moriarty P. 2018. Big Data for Urban Sustainability A HumanCentered Perspective. Berlin: Springer. 160

Williams R L, Stafford R, Goodenough A E. 2015. Biodiversity in urban gardens: Assessing the accuracy of citizen science data on garden hedgehogs. Urban Ecosyst, 18: 819-833

Wu J. 2014. Urban ecology and sustainability: The state-of-the-science and future directions. Landscape Urban Plan, 125: 209-221

Wu J, Xiang W, Zhao J. 2014. Urban ecology in China: Historical developments and future directions. Landscape Urban Plan, 125: 222-233

Xu Y, Belyi A, Santi P, Ratti C. 2019. Quantifying segregation in an integrated urban physical-social space. J R Soc Interface, 16: 20190536

Yang J, Zhao L, Mcbride J, Gong P. 2009. Can you see green? Assessing the visibility of urban forests in cities. Landscape Urban Plan, 91: 97-104

Ye T, Zhao N, Yang X, Ouyang Z, Liu X, Chen Q, Hu K, Yue W, Qi J, Li Z, Jia P. 2019. Improved population mapping for China using remotely sensed and points-of-interest data within a random forests 
model. Sci Total Environ, 658: 936-946

Yeow L W, Cheah L. 2019. Using spatially explicit commodity flow and truck activity data to map urban material flows. J Ind Ecol, 23: 1121-1132

Young R F, Wolf S A. 2006. Goal attainment in urban ecology research: A bibliometric review 1975-2004. Urban Ecosyst, 9: 179-193
Zhang F, Wu L, Zhu D, Liu Y. 2019. Social sensing from street-level imagery: A case study in learning spatio-temporal urban mobility patterns. ISPRS J Photogramm Remote Sens, 153: 48-58

Zhang X, Sun Y, Zheng A, Wang Y. 2020. A new approach to refining land use types: Predicting point-of-interest categories using Weibo check-in data. ISPRS Int J Geo-Inf, 9: 124

(责任编委: 李新) 